Bull. Austral. Math. Soc.

Vol. 41 (1990) [255-269]

\title{
HEREDITARY AND STRICT DOMAINS FOR RADICAL CLASSES OF ASSOCIATIVE RINGS
}

\author{
N.R. MCCONNELL
}

We generalise some existing results on strict radical classes, and introduce some new notions of hereditariness of radical classes. This is achieved by considering the classes of rings for which a radical class is hereditary and/or strict (in the general sense).

\section{INTRODUCTION}

It is well-known that a radical $\mathcal{R}$ is hereditary if and only if, for any ideal $I$ of any ring $A$, the equality $\mathcal{R}(I)=I \cap \mathcal{R}(A)$ holds. The analogous condition for left ideals, however, holds only very rarely (Gardner, [7]). This paper is concerned with conditions like this for other types of subrings. Since such equalities would not be expected to hold for all rings very often, we look at the classes of rings for which they do hold (for a given $\mathcal{R}$ ).

In Section 1 the basic constructions are given, which are used in Section 2 to build a theory for $\dashv$-strictness. This results in a generalisation of Stewart's work on strict radicals [12], and in determining the smallest $\dashv$-strict radical class containing a given radical class (see Gardner [6]). The third section investigates the constructions when the subrings considered are ideals, and arising from this some investigation is done into the question of whether there exists a ring in which no ideal has a simple homomorphic image. Finally, two detailed examples are given, showing some of the uses of the constructions. For radical-theoretic terminology and notation, we refer the reader to [14].

\section{Preliminaries}

Let $\dashv$ denote a type of distinguished subring satisfying

(I) $I \unlhd R \Rightarrow I \dashv R$,

(Ia) $S \dashv R, S \subseteq T \subseteq R \Rightarrow S \dashv T$,

(II) $S \dashv R \Leftrightarrow S^{\star} \dashv R / I$ where $S^{\star}=S / I, I \subseteq S, I \unlhd R$,

(III) $S+I \dashv R$ if $S \dashv R I \unlhd R$ and

(IIIa) $S \dashv R, T \dashv R \Rightarrow S \cap T \dashv R$.

Received 24 April, 1989

The author would like to express his sincere thanks to Dr. Barry Gardner, whose encouragement, ideas and assistance have played a major role in the production of this paper.

Copyright Clearance Centre, Inc. Serial-fee code: 0004-9729/90 \$A2.00+0.00. 
Some examples of subrings satisfying these are one-sided ideals, $(m, n)$-ideals [9], accessible subrings, meta-ideals [8], and subrings.

For any radical $\mathcal{R}$ we define the following two related classes:

$$
\begin{aligned}
\mathcal{K}_{1}(\mathcal{R}, \dashv) & =\{A \mid S \in \mathcal{R} \& S \dashv A \Rightarrow S \subseteq \mathcal{R}(A)\} \\
\text { and } \mathcal{K}_{2}(\mathcal{R}, \dashv) & =\{A \mid S \dashv A \Rightarrow \mathcal{R}(S)=S \cap \mathcal{R}(A)\} .
\end{aligned}
$$

Immediately we see that if $A \in \mathcal{K}_{2}(\mathcal{R}, \dashv), S \dashv A$ and $S \in \mathcal{R}$ then $S=\mathcal{R}(S)=$ $S \cap \mathcal{R}(A)$, so $S \subseteq \mathcal{R}(A)$; that is;

$$
\mathcal{K}_{2}(\mathcal{R},-1) \subseteq \mathcal{K}_{1}(\mathcal{R},-1)
$$

Before proceeding, we will prove

Leмma 1.1. For any radical classe $\mathcal{R}$, and any ring $A$, if $I \unlhd A$ and $I \subseteq \mathcal{R}(A)$, then $\mathcal{R}(A) / I=\mathcal{R}(A / I)$.

Proof: $\mathcal{R}(A) / I \in \mathcal{R}$ and $\mathcal{R}(A) / I \unlhd A / I$, so $\mathcal{R}(A) / I \subseteq \mathcal{R}(A / I)$. Also, $(A / I) /(\mathcal{R}(A) / I) \cong A / \mathcal{R}(A)$, so $\mathcal{R}[(A / I) /(\mathcal{R}(A) / I)]=0$, and thus $\mathcal{R}(A) / I \supseteq$ $\mathcal{R}(A / I)$.

We shall now characterise rings in the class $\mathcal{K}_{1}(\mathcal{R},-1)$ in terms of their semisimple images.

PROPOSITION 1.2. The following are equivalent:

(i) $A / I \in \mathcal{K}_{1}=\mathcal{K}_{1}(\mathcal{R},-1)$ for some $I \subseteq \mathcal{R}(A)$;

(ii) $A / \mathcal{R}(A) \in \mathcal{K}_{1}$;

(iii) $A \in \mathcal{K}_{1}$;

Proof: (i) $\Rightarrow$ (iii): Suppose $A / I \in \mathcal{K}_{1}$, where $I \subseteq \mathcal{R}(A), I \unlhd A$ and suppose $S \in \mathcal{R}$ and $S \dashv A$. Then by properties (II) and (III),

$$
\mathcal{R} \ni S /(S \cap I) \cong(S+I) / I \dashv \mathcal{R}(A / I) \text {. }
$$

By Lemma 1.1, $(S+I) \dashv \mathcal{R}(A) / I$, so $S+I \dashv \mathcal{R}(A)$, by property (II). Thus $S \subseteq \mathcal{R}(A)$; that is $A \in \mathcal{K}_{1}$ whenever $A / I \in \mathcal{K}_{1}$.

(iii) $\Rightarrow$ (ii): If $A \in \mathcal{K}_{1}, S^{\star} \dashv A / \mathcal{R}(A)$, then, by property (II), $S^{\star}=S / \mathcal{R}(A)$ for some $S \dashv A$ with $\mathcal{R}(A) \subseteq S$. Thus, if $S^{\star} \in \mathcal{R}, S / \mathcal{R}(A) \in \mathcal{R}$, so $S \in \mathcal{R}$; but then $S \subseteq \mathcal{R}(A)$, as $A \in \mathcal{K}_{1}$, so $S=\mathcal{R}(A)$. Hence, $S^{\star}=0=\mathcal{R}(A / \mathcal{R}(A))$; that is, $A / \mathcal{R}(A) \in \mathcal{K}_{1}$ whenever $A \in \mathcal{K}_{1}$.

(ii) $\Rightarrow$ (i) is clear.

Thus $\mathcal{K}_{1}(\mathcal{R},-1)$ is completely determined by its intersections with the semisimple class $\mathcal{S}$ of $\mathcal{R}$. We therefore define

$$
\begin{aligned}
\overline{\mathcal{K}}_{1}(\mathcal{R}, \dashv) & =\mathcal{S} \cap \mathcal{K}_{1}(\mathcal{R},-1) \\
& =\{A \mid \mathcal{R}(A)=0 ; \quad S \in \mathcal{R}, \quad S \dashv A \Rightarrow S=0\} .
\end{aligned}
$$


It then follows that any ring in $\mathcal{K}_{1}(\mathcal{R},-1)$ is an extension of an $\mathcal{R}$-radical ring by a ring of $\overline{\mathcal{K}}_{1}(\mathcal{R},-1)$, and these are precisely the $\mathcal{K}_{1}(\mathcal{R},-1)$ rings.

We now introduce two further properties that $\dashv$ may satisfy, one of which involves the particular radical class $\mathcal{R}$ being considered.

(IV) $S \dashv A \Rightarrow \mathcal{R}(S) \dashv A$.

(V) $S \dashv I \unlhd A \Rightarrow S \dashv A$.

Note that if $S \dashv T, T \dashv A \Rightarrow S \dashv A$, both of these properties are satisfied, and that if $S \dashv A$ means $S \unlhd A$, property (IV) is simply the Anderson-Divinsky-Sulinski property.

If $\mathcal{R}, \dashv$ satisfy property (IV), then

$$
\begin{aligned}
\overline{\mathcal{K}}_{1}(\mathcal{R}, \dashv) & =\{A \mid \mathcal{R}(A)=0 \& S \dashv A \Rightarrow \mathcal{R}(S)=0\} \\
& =\{A \mid S \dashv A \Rightarrow \mathcal{R}(S)=0\} .
\end{aligned}
$$

This will be used later.

From property (V) we get the following:

Proposition 1.3. If $S \dashv I \unlhd A \Rightarrow S \dashv A$, then $\overline{\mathcal{K}}_{1}(\mathcal{R}, \dashv)$ is a semisimple class.

Proof: Let $I \unlhd A ; I, A / I \in \overline{\mathcal{K}}_{1}=\overline{\mathcal{K}}_{1}(\mathcal{R}, \dashv) ; S \dashv A, S \in \mathcal{R}$. Then

$$
\mathcal{R} \ni S /(S \cap I) \cong(S+I) / I \dashv A / I \text {. }
$$

Thus $S /(S \cap I)=0$; that is $S \subseteq I$, and so by property (Ia), $S \dashv I$. But then $S=0$, so $A \in \overline{\mathcal{K}}_{1}$ and $\overline{\mathcal{K}}_{1}$ is closed under extensions.

Let $A$ be a subdirect product of $\left\{A_{\lambda} \mid \lambda \in \Lambda\right\}, A_{\lambda} \cong A / I_{\lambda}$ for all $\lambda, A_{\lambda} \in \overline{\mathcal{K}}_{1}$ for all $\lambda$. If $S \nmid A, S \in \mathcal{R}$, then

$$
\mathcal{R} \ni S /\left(S \cap I_{\lambda}\right) \cong\left(S+I_{\lambda}\right) / I_{\lambda}
$$

for all $\lambda$, so $S /\left(S \cap I_{\lambda}\right)=0$ for all $\lambda$ and thus $S$ is contained in every $I_{\lambda}$. But $\bigcap_{\lambda} I_{\lambda}=0$, so $S=0$ and $A \in \overline{\mathcal{K}}_{1}$; that is $\overline{\mathcal{K}}_{1}$ is closed under subdirect products.

Let $I \unlhd A \in \overline{\mathcal{K}}_{1}$, and $S \dashv I, S \in \mathcal{R}$. Then, by hypothesis, $S \dashv A$, so $S=0$ and $\overline{\mathcal{K}}_{1}$ is hereditary. That is, $\overline{\mathcal{K}}_{1}(\mathcal{R},-1)$ is a semisimple class.

We now turn our attention to $\mathcal{K}_{2}(\mathcal{R},-1)$; recall that

$$
\mathcal{K}_{2}=\mathcal{K}_{2}(\mathcal{R}, \dashv)=\{A \mid S \dashv A \Rightarrow \mathcal{R}(S)=S \cap \mathcal{R}(A)\} .
$$

Proposition 1.4.

(i) If $A \in \mathcal{K}_{2}$, then $A / \mathcal{R}(A) \in \mathcal{K}_{2}$.

(ii) If $\mathcal{R}(A) \in \mathcal{K}_{2}$ and $A / \mathcal{R}(A) \in \mathcal{K}_{2}$, then $A \in \mathcal{K}_{2}$. 
Proof: (i) Suppose $A \in \mathcal{K}_{2}$, and let $S^{\star} \dashv A / \mathcal{R}(A)$. Then $S^{\star}=S / \mathcal{R}(A)$ for some $S \dashv A, \mathcal{R}(A) \subseteq S$ by (II), so $\mathcal{R}(S)=S \cap \mathcal{R}(A)$ as $A \in \mathcal{K}_{2}$. But then $\mathcal{R}(S)=$ $\mathcal{R}(A)$, so $S^{\star}=S / \mathcal{R}(A)$ is $\mathcal{R}$-semisimple, and $\mathcal{R}\left(S^{\star}\right)=0=S^{\star} \cap \mathcal{R}(A / \mathcal{R}(A))$; that is, $A / \mathcal{R}(A) \in \mathcal{K}_{2}$.

(ii) Let $A$ be a ring with $A / \mathcal{R}(A), \mathcal{R}(A) \in \mathcal{K}_{2}$. Let $S \dashv A$; then

$$
\mathcal{R}((S+\mathcal{R}(A)) / \mathcal{R}(A))=((S+\mathcal{R}(A)) / \mathcal{R}(A)) \cap \mathcal{R}(A / \mathcal{R}(A))=0
$$

and $\mathcal{R}(S \cap \mathcal{R}(A))=S \cap \mathcal{R}(A) \cap \mathcal{R}(\mathcal{R}(A))=S \cap \mathcal{R}(A)$ since $S \cap \mathcal{R}(A) \dashv \mathcal{R}(A)$ by (Ia) and (IIIa). But $(S+\mathcal{R}(A)) / \mathcal{R}(A) \cong S /(S \cap \mathcal{R}(A))$, so $\mathcal{R}(S /(S \cap \mathcal{R}(A)))=0$, and $\mathcal{R}(S)=S \cap \mathcal{R}(A)$; that is $A \in \mathcal{K}_{2}$.

Now, analogously to our treatment of $\mathcal{K}_{1}(\mathcal{R},-1)$, we look at the class

$$
\begin{aligned}
\overline{\mathcal{K}}_{2}(\mathcal{R}, \dashv) & =\mathcal{S} \cap \mathcal{K}_{2}(\mathcal{R}, \dashv) \\
& =\{A \mid S \dashv A \Rightarrow \mathcal{R}(S)=0\},
\end{aligned}
$$

and note that if $\mathcal{R}, \dashv$ satisfy property (IV), then this is equal to $\overline{\mathcal{K}}_{1}(\mathcal{R},-1)$.

We also have, as with $\overline{\mathcal{K}}_{1}(\mathcal{R},-1)$,

Proposition 1.5. If $S \dashv I \unlhd A \Rightarrow S \dashv A$, then $\overline{\mathcal{K}}_{2}(\mathcal{R}, \dashv)$ is a semisimple class.

Proof: Let $I \unlhd A$ and $I, A / I \in \overline{\mathcal{K}}_{2}=\overline{\mathcal{K}}_{2}(\mathcal{R}, \dashv)$. Then $\mathcal{R}(A)=0$. Let $S \dashv A$; then

$$
S /(S \cap I) \cong(S+I) / I \dashv A / I,
$$

so $\mathcal{R}(S /(S \cap I))=0$ as $A / I \in \overline{\mathcal{K}}_{2}$. Now $S \cap I \dashv I$ (by (Ia) and (IIIa)), so $\mathcal{R}(S \cap I)=0$ (as $I \in \overline{\mathcal{K}}_{2}$ ). But then $\mathcal{R}(S)=0$, so $A \in \overline{\mathcal{K}}_{2}$; that is, $\overline{\mathcal{K}}_{2}$ is closed under extensions.

Let $A$ be a subdirect product of $\left\{A_{\lambda} \mid \lambda \in \Lambda\right\}, A_{\lambda} \cong A / I_{\lambda}$ for all $\lambda, A_{\lambda} \in \overline{\mathcal{K}}_{2}$ for all $\lambda$. Then $\mathcal{R}(A)=0$. Let $S \dashv A$; then for any $\lambda,\left(I_{\lambda}+S\right) / I_{\lambda} \dashv A_{\lambda}$ so $\mathcal{R}\left(\left(I_{\lambda}+S\right) / I_{\lambda}\right)=0$. Hence, for any $\lambda, \mathcal{R}\left(S /\left(I_{\lambda} \cap S\right)\right)=0$, so $\mathcal{R}(S) \subseteq I_{\lambda} \cap S \subseteq I_{\lambda}$ and thus $\mathcal{R}(S) \subseteq \bigcap_{\lambda} I_{\lambda}=0$. That is, $A \in \overline{\mathcal{K}}_{2}$, and $\overline{\mathcal{K}}_{2}$ is closed under subdirect products.

Let $I \unlhd A \in \overline{\mathcal{K}}_{2}$, and let $S \dashv I$. Then, by hypothesis, $S \dashv A$, so $\mathcal{R}(S)=0$; that is, $\overline{\mathcal{K}}_{2}$ is hereditary (as clearly $\mathcal{R}(I)=0$ ). Hence, $\overline{\mathcal{X}}_{2}(\mathcal{R},-1$ ) is a semisimple class.

We define a class $\mathcal{R}$ to be $\dashv$-hereditary if $S \in \mathcal{R}$ whenever $S \dashv A \in \mathcal{R}$. Now, for a radical class $\mathcal{R}$, consider the rings $A$ for which $S \in \mathcal{R}$ whenever $S \dashv A$. We have

$$
\begin{aligned}
\{A \mid A \in \mathcal{R} \& S \dashv A \Rightarrow S \in \mathcal{R}\} & =\{A \mid A \in \mathcal{R} \& S \dashv A \Rightarrow \mathcal{R}(S)=S \cap \mathcal{R}(A)\} \\
& =\mathcal{K}_{2}(\mathcal{R}, \dashv) \cap \mathcal{R} .
\end{aligned}
$$

This leads to the following: 
THEOREM 1.6. If $S \dashv A \Rightarrow \mathcal{R}(S) \dashv A$, then the following are equivalent:

(i) $\mathcal{R}$ is t-hereditary;

(ii) $\mathcal{R} \subseteq \mathcal{K}_{2}(\mathcal{R},-1)$;

(iii) $\mathcal{K}_{1}(\mathcal{R},-1)=\mathcal{K}_{2}(\mathcal{R},-1)$;

Proof: (i) $\Leftrightarrow$ (ii):

$$
\{A \mid A \in \mathcal{R} \& S \dashv A \Rightarrow S \in \mathcal{R}\}=\mathcal{K}_{2}(\mathcal{R}, \dashv) \cap \mathcal{R}
$$

(i) means that the left side of this equation is equal to $\mathcal{R}$; (ii) means that the right side is.

(iii) $\Rightarrow$ (ii):

$$
\mathcal{R} \subseteq \mathcal{K}_{1}(\mathcal{R},-1)
$$

(ii) $\Rightarrow$ (iii): From the comments preceding Proposition 1.5, $A / \mathcal{R}(A) \in \overline{\mathcal{K}}_{1}$ for a ring $A$ if and only if $A / \mathcal{R}(A) \in \overline{\mathcal{K}}_{2}$.

By (ii), $\mathcal{R}(A) \in \mathcal{K}_{2}$ for any ring $A$, so Proposition 1.4 becomes $A \in \mathcal{K}_{2}$ if and only if $A / \mathcal{R}(A)$. Thus we have (by Proposition 1.2)

$$
A \in \mathcal{K}_{2} \Leftrightarrow A / \mathcal{R}(A) \in \overline{\mathcal{K}}_{2} \Leftrightarrow A / \mathcal{R}(A) \in \overline{\mathcal{X}}_{1} \Leftrightarrow A \in \mathcal{K}_{1}
$$

that is, $\mathcal{K}_{1}(\mathcal{R},-1)=\mathcal{K}_{2}(\mathcal{R},-1)$.

Note also that under these conditions, $\mathcal{K}_{2}(\mathcal{R},-1)$ is exactly the class of $\mathcal{R}$-rings extended by $\overline{\mathcal{K}}_{2}(\mathcal{R},-1)$-rings.

COROLLARY 1.7. A radical class $\mathcal{R}$ is strongly hereditary and strict if and only if $S \cap \mathcal{R}(A)=\mathcal{R}(S)$ for every subring $S$ of $A$.

Proof: Let $S \dashv A$ mean " $S$ is a subring of $A$ ". Clearly $S \dashv A$ implies that $\mathcal{R}(S) \dashv A$, and $\mathcal{K}_{1}(\mathcal{R},-1)$ is the class of all rings if and only if $\mathcal{R}$ is strict.

Thus if $\mathcal{R}$ is strongly hereditary and stict, $\mathcal{K}_{1}(\mathcal{R},-1)=\mathcal{K}_{2}(\mathcal{R},-1)$ is the class of all rings (since strongly hereditary means †-hereditary). That is, $\mathcal{R}(S)=S \cap \mathcal{R}(A)$ for all $A, S \dashv A$.

Conversely, if $\mathcal{R}(S)=S \cap \mathcal{R}(A)$ for all $A, S \dashv A$, then $\mathcal{K}_{2}(\mathcal{R},-1)$ is the class of all rings. But $\mathcal{K}_{2}(\mathcal{R},-1) \subseteq \mathcal{K}_{1}(\mathcal{R},-1)$, so $\mathcal{K}_{1}(\mathcal{R},-1)=\mathcal{K}_{2}(\mathcal{R},-1)$ is the class of all rings, and $\mathcal{R}$ is strongly hereditary and strict.

\section{2. †-STRICT CLOSURE}

We now use the results of the previous section to generalise Section 2 of Stewart's paper on strict radical classes [12]. This section will be largely patterned on Stewart's work.

Define a radical class $\mathcal{R}$ to be - -strict if $\mathcal{K}_{1}(\mathcal{R},-1)$ is the class of all rings (so that if $\dashv$ denotes "is a subring of", being - -strict is the same as being strict). 
THEOREM 2.1. If $\mathcal{R}, \dashv$ satisfy property (IV), then $\mathcal{R}$ is $\dashv$-strict if and only if its semisimple class $\mathcal{S}$ is t-hereditary.

Proof: Let $\mathcal{R}$ be a $\dashv$-strict radical class, and let $S \dashv A \in S$. Then, by (IV), $\mathcal{R}(S) \dashv A$, so by $\dashv$-strictness $\mathcal{R}(S) \subseteq \mathcal{R}(A)=0$. Hence, $S \in \mathcal{S}$, and $\mathcal{S}$ is †-hereditary.

Conversely, let $S$ be a $\dashv$-hereditary semisimple class, and let $S \dashv A$ with $S \in \mathcal{R}$. Then

$$
\mathcal{R} \ni S /(S \cap \mathcal{R}(A)) \cong(S+\mathcal{R}(A)) / \mathcal{R}(A) \dashv A / \mathcal{R}(A) \in \mathcal{S},
$$

so $S=S \cap \mathcal{R}(A)$; that is $S \subseteq \mathcal{R}(A)$ and thus $\mathcal{R}$ is a $\dashv$-strict radical class.

If $\mathcal{R}, \dashv$ satisfy properties (IV) and (V), which we assume from now on, then by Proposition 1.5 and the remarks before it, $\overline{\mathcal{K}}_{1}=\overline{\mathcal{K}}_{2}$ is a semisimple class; we now proceed to characterise the radical class corresponding to this semisimple class.

Let $\mathcal{C}$ be a class of rings, and denote by $\mathcal{C}_{\dashv}$ the class of all rings $R$ such that for every non-zero homomorphic image $A$ of $R$ there exists $0 \neq S \dashv A$ such that $S$ is isomorphic to a ring in $\mathcal{C}$. It follows from [4, Theorem 1] that $\mathcal{C}_{-}$is a radical class.

THEOREM 2.2. A radical class $\mathcal{R}$ is $\dashv$-strict if and only if $\mathcal{R}=\mathcal{C}_{\dashv}$ for some class $\mathcal{C}$ of rings.

PROoF: If $\mathcal{R}$ is a $\dashv$-strict radical class, then $\mathcal{R}=\mathcal{R}_{\dashv}$.

For the converse, let $\mathcal{C}$ be any class of rings, $A$ be a ring, $S \dashv A$. Then $\bar{S}=$ $S+A S+S A+A S A$ is the ideal of $A$ generated by $S$. We need only to prove that if $S \in \mathcal{C}_{-1}$, then $\bar{S} \in \mathcal{C}_{-1}$, for it then follows that $S \subseteq \mathcal{C}_{-1}(A)$.

Let $\bar{S} / K$ be an arbitrary non-zero homomorphic image of $\bar{S}$. The result follows if we can find a non-zero homomorphism $f: S \rightarrow \bar{S} / K$ such that $f(S) \dashv \bar{S} / K$.

If $S \nsubseteq K$, then the function defined by $x \rightarrow x+K$ for $x \in S$ is as required, since $S+K / K \dashv \bar{S} / K$.

If $S \subseteq K$, then by Andrunakievic's lemma [1, Lemma 4] we have $\bar{S}^{3} \subseteq K$. Choose $n \geqslant 2$ to be minimal with respect to $\bar{S}^{n} \subseteq K$. Then $\bar{S}^{n-1} \nsubseteq K$, so $(S+A S) \bar{S}^{n-2} \nsubseteq K$ (for the case $n=2$, take $\bar{S}^{n-2}$ to be the ring $A^{\star}$ obtained by adjoining an identity to $A$ ). Now choose $m \in Z, a \in A, b \in \bar{S}^{n-2}$ such that $(m+a) S b \nsubseteq K$, and let $f: S \rightarrow S / K$ be defined by

$$
f(x)=(m+a) x b+K .
$$

Clearly $f$ is a well-defined, non-zero additive homomorphism. Also, for $x, y \in S$, we have

$$
(m+a) x y b \in A^{\star} S^{2} \bar{S}^{n-2} \subseteq \bar{S} \subseteq K
$$

and

$$
(m+a) x b(m+a) y b \in A^{\star} S \bar{S}^{n-2} A^{\star} S \bar{S}^{n-2} \subseteq \bar{S}^{n} \subseteq K .
$$

That is,

$$
f(x y)=f(x) f(y)=0 .
$$


Thus $f$ is a non-zero homomorphism. Finally, $\bar{S} / K$ is nilpotent, so by [3, Proposition 8] every subring of $\bar{S} / K$ is accessible. Thus $f(S)$ is an accessible subring of $\bar{S} / K$, and by repeated application of $(\mathrm{V}), f(S) \dashv \bar{S} / K$ as required.

Corollary 2.3. Let $\mathcal{C}$ be a class of rings, and let $A$ be any ring.

(i) If $\mathcal{C}$ is homomorphically closed, then $A$ is $\mathcal{C}_{-1}$-semisimple if and only if $S \notin \mathcal{C}$ for all non-zero $S \dashv A$.

(ii) If $\mathcal{C}$ is a radical class, then $A$ is $\mathcal{C}_{-}$-semisimple if and only if $\mathcal{C}(S)=0$ for all $S \dashv A$.

ProOF: (i) is true by property (V), and (ii) follows by property (IV).

Corollary 2.4. A radical class is t-strict if and only if it the upper radical class determined by a †-hereditary class of rings.

PROOF: If $\mathcal{L}$ is a $\dashv$-hereditary (and hence hereditary) class of rings, then the upper radical class determined by $\mathcal{L}$ is the class of all rings which have no homomorphic image in $\mathcal{L}$. If $\mathcal{C}$ is the class of all rings not in $\mathcal{L}$, then $\mathcal{C}_{\dashv}$ is the class of all rings $A$ such that for every non-zero homomorphic image $A^{\star}$ of $A$, there exists $0 \neq S \dashv A^{\star}$ with $S$ not in $\mathcal{L}$. That is, $\mathcal{C}_{\dashv}$ is the upper radical class determined by $\mathcal{L}$, since $\mathcal{L}$ is $\dashv$-hereditary. The converse is immediate from Theorem 2.1

We are now in a position to prove the main result in this Section.

TheOREM 2.5. Let $\mathcal{R}$ be a radical class, $\dashv$ a subring property satisfying (IV) and $(V)$. Then $\mathcal{R}_{\dashv}$ is the smallest $\dashv$-strict radical class containing $\mathcal{R}$, and $\overline{\mathcal{K}}_{1}(\mathcal{R}, \dashv)=$ $\overline{\mathcal{X}}_{2}(\mathcal{R},-1)$ is the corresponding semisimple class.

Proof: Clearly $\mathcal{R} \subseteq \mathcal{R}_{\dashv}$, and $\mathcal{R}_{\dashv}$ is a $\dashv$-strict radical class. By ( $\star$ ) and Corollary 2.3(ii), $\overline{\mathcal{K}}_{1}(\mathcal{R},-1)$ is the corresponding semisimple class.

Let $\mathcal{S}$ be the semisimple class corresponding to $\mathcal{R}$, and let $\mathcal{M}$ be any $\dashv$-hereditary semisimple class contained in $\mathcal{S}$. If $S \dashv A \in \mathcal{M}$, then $S \in \mathcal{M}$, so $S \in \mathcal{S}$ and $\mathcal{R}(S)=0$. Thus $A \in \overline{\mathcal{K}}_{1}(\mathcal{R},-1)$; that is, $\mathcal{M}$ is contained in $\overline{\mathcal{K}}_{1}(\mathcal{R},-1)$. This means that $\overline{\mathcal{K}}_{1}(\mathcal{R},-1)$ is the largest $\dashv$-hereditary semisimple class contained in $\mathcal{S}$, and it follows by Theorem 2.1 and $\left[14\right.$, Theorem 7.2] that $\mathcal{R}_{\dashv}$ is the smallest $\dashv$-strict radical class containing $\mathcal{R}$. 0

We now investigate the nature of $\mathcal{R}_{-}(A)$ for a given ring $A$. We define the following distinguished subring of $A$ :

$$
\mathcal{K}_{1}^{\star}(A)=\langle S \dashv A \mid S \in \mathcal{R}\rangle
$$

where $\left\langle\left\{S_{i}\right\}\right\rangle$ denotes the subring generated by the $S_{i}$. Then $\mathcal{K}_{1}^{\star}(A)=0$ if and only if $A \in \overline{\mathcal{K}}_{1}(\mathcal{R}, \dashv)$, and from property (IV), $\mathcal{K}_{1}^{\star}(A)=\langle\mathcal{R}(S) \mid S \dashv A\rangle$. Now if $S \dashv A$, $S \in \mathcal{R}$,

$$
\mathcal{R} \ni S /\left(S \cap \mathcal{R}_{\dashv}(A)\right) \cong\left(S+\mathcal{R}_{\dashv}(A)\right) / \mathcal{R}_{\dashv}(A) \in \overline{\mathcal{K}}_{1}(\mathcal{R}, \dashv)
$$

so $S=S \cap \mathcal{R}_{\dashv}(A)$ and $S \subseteq \mathcal{R}_{\dashv}(A)$. Thus $\mathcal{K}_{1}^{\star}(A) \subseteq \mathcal{R}_{\dashv}(A)$. 
Question. Is $\mathcal{R}_{\dashv}(A)$ the ideal of $A$ generated by $\mathcal{K}_{1}^{\star}(A)$ ?

\section{HEREDitaRINESS AND $\mathcal{K}_{2}(\mathcal{R}, \unlhd)$}

In this section, we will look at the case where $A \dashv B$ means $A$ is an ideal of $B$. In this case, the class $\mathcal{K}_{1}(\mathcal{R}, \unlhd)$ is simply the class of all rings, but $\mathcal{K}_{2}(\mathcal{R}, \unlhd)$ is the class of all rings only if $\mathcal{R}$ is hereditary. The extent of $\mathcal{K}_{2}(\mathcal{R}, \unlhd)$ can be thought of as, in some sense, a measure of the "hereditariness" of $\mathcal{R}$. We therefore look at the possibilities for $\mathcal{K}_{2}(\mathcal{R}, \unlhd)$ when $\mathcal{R}$ is not hereditary.

Let $\mathcal{R}$ be any radical class, and $\mathcal{S}$ its semisimple class. Then $\mathcal{S}$ is contained in $\mathcal{K}_{2}(\mathcal{R}, \unlhd)$, for if $A$ is any ring in $\mathcal{S}, I$ an ideal of $A$, then $\mathcal{R}(I)=0=I \cap \mathcal{R}(A)$, so $A \in \mathcal{K}_{2}(\mathcal{R}, \unlhd)$.

The following example demonstrates that $\mathcal{S}$ can be equal to $\mathcal{K}_{2}(\mathcal{R}, \unlhd)$.

Let $\mathcal{R}$ denote the class of torsion divisible rings, and $\mathcal{S}$ its semisimple class. If $A \notin \mathcal{S}$, let $A_{p}=\{x \in \mathcal{R}(A) \mid p x=0\}$; then $A_{p} \neq 0$ for some prime $p$, and $A_{p}$ is a subring of $A$. For $x \in A_{p}$ and $a \in A$ we have

$$
p(x a)=(p x) a=0 \text { and } p(a x)=a(p x)=0
$$

and, since $x a$ and $a x$ are in $\mathcal{R}(A)$, they are in $A_{p}$ also. That is, $A_{p}$ is an ideal of $A$. But $A_{p}$ is not $p$-divisible, so $A_{p} \notin \mathcal{R}$. Thus $A_{p} \unlhd A$, and $\mathcal{R}\left(A_{p}\right) \neq A_{p} \cap \mathcal{R}(A)=A_{p}$. That is, $\notin \mathcal{K}_{2}(\mathcal{R}, \unlhd)$ and hence $\mathcal{K}_{2}(\mathcal{R}, \unlhd)=\mathcal{S}$.

Another measure of the "hereditariness" of a radical class is the size of its largest hereditary radical subclass; relating to this we have

Proposition 3.1. Let $\mathcal{R}$ be an hereditary radical class containing no simple rings. Then $\mathcal{R}=\{0\}$.

Proof: Let $A^{2}=0$. Then if $\mathcal{R}(A) \neq 0, \mathcal{R}(A)$ has an ideal isomorphic to the zero-ring on a prime field $\left(Z_{p}^{0}\right)$ or on the integers $\left(Z^{0}\right)$. But if $Z^{0} \cong I \subseteq \mathcal{R}(A)$, then $Z^{0} \in \mathcal{R}$ and so $Z_{p}^{0} \in \mathcal{R}$ for any prime $p$. Either way, $\mathcal{R}$ contains a simple zeroring-a contradiction, so $\mathcal{R}(A)=0$ for any zero-ring $A$. This means every ring in $\mathcal{R}$ is idempotent, and so hereditarily idempotent, since $\mathcal{R}$ is hereditary. Thus every homomorphic image of an $\mathcal{R}$-ring is semiprime.

Suppose that there exists a non-zero $\mathcal{R}$-ring, $R$. Then $R$ has an homomorphic image $\bar{R}$ which is subdirectly irreducible. Also, $\bar{R}$ is semiprime, so the heart $H$ of $\bar{R}$ is simple. But $\bar{R} \in \mathcal{R}$, so $H \in \mathcal{R}$ as $\mathcal{R}$ is hereditary-a contradiction as $\mathcal{R}$ contains no simple rings. Thus there exists no such $R$, and $\mathcal{R}=\{0\}$.

The author is indebted to Dr. B.J. Gardner for this proof.

TheOREM 3.2. The following are equivalent for a radical class $\mathcal{R}$.

(i) Every $\mathcal{R}$-ring has no maximal ideals. 
(ii) $\mathcal{R}$ contains no simple rings.

(iii) $\{0\}$ is the largest hereditary radical subclass of $\mathcal{R}$.

Proof: (i) $\Leftrightarrow$ (ii) If every $\mathcal{R}$-ring has no maximal ideals, then $\mathcal{R}$ contains no simple rings, as 0 is always maximal in a simple ring.

Conversely, if $\mathcal{R}$ contains no simple rings, then no $\mathcal{R}$-ring can contain a maximal ideal, since this would entail having a simple homomorphic image.

(ii) $\Leftrightarrow$ (iii) If $\mathcal{R}$ contains no simple rings, then nor does any hereditary radical class contained in $\mathcal{R}$. It follows by Proposition 3.1 that any such class must be $\{0\}$.

Conversely, if $\mathcal{R}$ is a radical class containing no non-zero hereditary radical classes, then $\mathcal{R}$ contains no simple rings, as the lower radical class determined by a simple ring is hereditary.

Relating this back to $\mathcal{K}_{2}(\mathcal{R}, \unlhd)$, we have

Proposition 3.3. Each of the following conditions on a radical class $\mathcal{R}$ and its semisimple class $\mathcal{S}$ implies its successor:

(i) $\mathcal{K}_{2}(\mathcal{R}, \unlhd)=\mathcal{S}$;

(ii) $\mathcal{K}_{2}(\mathcal{R}, \unlhd) \cap \mathcal{R}=\{0\}$;

(iii) $\{0\} \neq \mathcal{R}^{\prime} \subseteq \mathcal{R} \Rightarrow \mathcal{R}^{\prime} \nsubseteq \mathcal{K}_{2}(\mathcal{R}, \unlhd)$ where $\mathcal{R}^{\prime}$ is a radical class;

(iv) $\{0\}$ is the largest hereditary radical subclass of $\mathcal{R}$.

Proof: (i) implies (ii) and (ii) implies (iii) are clear. Suppose that (iii) holds, and let $\mathcal{R}^{\prime}$ be an hereditary radical subclass of $\mathcal{R}$. Then $\mathcal{R}^{\prime} \nsubseteq \mathcal{K}_{2}(\mathcal{R}, \unlhd)$ or $\mathcal{R}^{\prime}=\{0\}$. Suppose $\mathcal{R}^{\prime} \nsubseteq \mathcal{K}_{2}(\mathcal{R}, \unlhd)$, and choose $A \in \mathcal{R}^{\prime}$ with $A \notin \mathcal{K}_{2}(\mathcal{R}, \triangleleft)$. Then there is an ideal $I$ of $A$ with $\mathcal{R}(I) \neq I \cap \mathcal{R}(A)$. But $A \in \mathcal{R}^{\prime} \subseteq \mathcal{R}$, so $\mathcal{R}(I) \neq I$; that is, $I \notin \mathcal{R}$, and thus $I \notin \mathcal{R}^{\prime}$-a contradiction, as $\mathcal{R}^{\prime}$ is hereditary. Hence $\mathcal{R}^{\prime}=\{0\}$, and (iv) holds.

PROPOSITION 3.4. The equivalence of (iv) and (ii) is equivalent to the existence in every ring of an ideal with a simple homomorphic image.

Proof: Suppose that (iv) is equivalent to (ii). By [11], $\mathcal{R} \cap \mathcal{K}_{2}(\mathcal{R}, \unlhd)$ is a radical class $(\overline{\mathcal{R}}$ in the notation of [11]). Let $\mathcal{R}$ be the upper radical determined by the class of all simple rings. Then $\mathcal{R}$ contains no simple rings, so $\mathcal{R} \cap \mathcal{K}_{2}(\mathcal{R}, \unlhd)=\{0\}$. But

$$
\begin{aligned}
\mathcal{R} \cap \mathcal{K}_{2}(\mathcal{R}, \unlhd) & =\{A \in \mathcal{R} \mid I \unlhd A \Rightarrow \mathcal{R}(I)=I \cap \mathcal{R}(A)\} \\
& =\{A \in \mathcal{R} \mid I \unlhd A \Rightarrow I \in \mathcal{R}\} \\
& =\{A \mid 0 \neq I \Rightarrow I \text { has no simple image }\}
\end{aligned}
$$

Thus every non-zero ring has an ideal with a simple image.

Conversely, supposing such an ideal to exist in every ring, let $\mathcal{R}$ be any radical class containing no simple rings. For any non-zero ring $A$ in $\mathcal{K}_{2}(\mathcal{R}, \unlhd) \cap R, I \triangleleft A$ 
implies that $I \in \mathcal{R}$. But then if $I_{0}$ is an ideal which maps onto a simple ring $S$, we have $S \in \mathcal{R}$-a contradiction. Thus no such ring exists, and $\mathcal{K}_{2}(\mathcal{R}, \unlhd) \cap \mathcal{R}=\{0\}$.

We will thus investigate the properties of rings in $\mathcal{K}_{2}(\mathcal{R}, \unlhd) \cap \mathcal{R}$ where $\mathcal{R}$ is the upper radical of the class of simple rings, and write $\mathcal{K}_{2}(\mathcal{R}, \unlhd) \cap \mathcal{R}=\mathcal{R}_{0}$.

Let $A \in \mathcal{R}_{0}$. Then $A / A^{2} \in \mathcal{R}$, and thus $A / A^{2}=0$ as otherwise $A / A^{2}$ has an ideal isomorphic to $Z^{0}$ (which maps onto $Z_{p}^{0}$ ) or $Z_{p}^{0}$. Thus $\mathcal{R}_{0}$ consists of idempotent rings. If $R$ is a subdirectly irreducible $\mathcal{R}_{0}$-ring with heart $H$, then $H^{2}=0$, since $H$ cannot be idempotent, and $H^{+}$is divisible, as $H$ cannot map onto $Z_{p}^{0}$ for any $p$. If $p$ is a prime and $p A \neq A \in \mathcal{R}_{0}$, then $A / p A$ has subdirectly irreducible factors with characteristic $p$, a contradiction, as any subdirectly irreducible $\mathcal{R}_{0}$-ring has divisible heart. Thus every $\mathcal{R}_{0}$-ring is divisible.

Now, suppose that there is a subdirectly irreducible $\mathcal{R}_{0}$-ring $R$ with torsion heart $H$, so for some prime $p, H$ has a subring isomorphic to $\mathrm{Z}_{p}^{0}$. Let $a \in H, n \in \mathrm{Z}^{+}$ with $n a=0$, and let $b \in R$. Then, since $R$ is divisible, there exists $c \in R$ with $n c=b$, so $a b=n a c=0=n c a=b a$, and $H$ is contained in the annihilator of $R$. But then every subring of $H$ is an ideal of $R$, so $R$ has an ideal isomorphic to $\mathrm{Z}_{p}^{0}$-a contradiction. Thus all subdirectly irreducible $\mathcal{R}_{0}$-rings have torsion-free hearts, and consequently are torsion-free, since the heart must be contained in the largest torsion ideal if the latter is non-zero. Since every $\mathcal{R}_{\mathbf{0}}$-ring is a subdirect product of subdirectly irreducible $\mathcal{R}_{0}$-rings, every $\mathcal{R}_{0}$-ring is torsion-free.

We can also see that if $b$ is a non-zero element which annihilates an $\mathcal{R}_{0}$-ring $A$, then the ideal of $A$ generated by $b$ is isomorphic to $Z^{0}$ or $Z_{p}^{0}$, either of which maps onto a simple ring, and so no such $b$ can exist and $\mathcal{R}_{0}$-rings can have no annihilator.

Finally, every $\mathcal{R}_{0}$-ring has accessible subrings which are not ideals, since the heart of every subdirectly irreducible $\mathcal{R}_{0}$-ring has ideals which are not ideals of $R$.

To sum up, the equivalence of (ii) and (iv) in Proposition 3.3 depends on the nonexistence of a torsion-free, idempotent, divisible ring with zero annihilator in which not every accessible subring is an ideal.

\section{EXAMPLES}

Our first example is of a radical class usually regarded as being far from hereditary. The class of all rings with divisible additive groups is a radical class, which we will denote by $\mathcal{D}$. For any ring $A, \mathcal{D}(A)$ is the maximal divisible subgroup, which is always an ideal, by $[5, \mathrm{p} .278]$.

It is clear that $\mathcal{D}$ contains simple rings (the field $Q$ of rational numbers, for example), so $\mathcal{D}$ does not satisfy any of the conditions of Proposition 3.3. Now a ring $A$ is contained in $\mathcal{K}_{2}(\mathcal{D}, \triangleleft)$ if and only if $I \cap \mathcal{D}(A)$ is divisible for every ideal $I$ of $A$. Thus 
we have

$$
\begin{aligned}
\mathcal{K}_{2}(\mathcal{D}, \unlhd) & =\{A \mid(\forall I \unlhd A)(I \cap \mathcal{D}(A) \text { is divisible })\} \\
& =\{A \mid I \unlhd A \& I \subseteq \mathcal{D}(A) \Rightarrow I \in \mathcal{D}\} \\
& =\left\{A \mid I \subseteq \mathcal{D}(A) \& I \unlhd A \Rightarrow I^{+} \text {is a sum of full rational and quasicyclic groups }\right\}
\end{aligned}
$$

where $I^{+}$is the additive group of $I$.

Now, suppose the torsion part of $\mathcal{D}(A)$ is non-zero. Then the p-component of $\mathcal{D}(A)$ is non-zero for some prime $p$; call this component $K$. It follows that $K^{+}$is a sum of quasicyclic groups, and hence $K$ has trivial multiplication by [13, Theorem 2]. Let $K[p]=\{x \in K \mid p x=0\}$, and let $a \in A, s \in K[p]$. Then $s a$ and $a s$ are zero or have order $p$, so they are contained in $K[p]$ and thus $K[p] \unlhd A$. But $K[p] \notin \mathcal{D}$, so $\mathcal{D}(K[p]) \neq \mathcal{D}(A) \cap K[p]$ and $A \notin \mathcal{K}_{2}(\mathcal{D}, \unlhd)$. Thus we have

$$
\begin{aligned}
\mathcal{K}_{2}(\mathcal{D}, \unlhd)= & \{A \mid I \subseteq \mathcal{D}(A) \& I \unlhd A \Rightarrow \\
& \left.I^{+} \text {is a sum of full rational groups }\right\} \\
= & \{A \mid \mathcal{D}(A) \text { is a Q-algebra; } I \subseteq \mathcal{D}(A) \& I \unlhd A \Rightarrow \\
& I \text { is an algebra ideal of } \dot{\mathcal{D}}(A)\} .
\end{aligned}
$$

Finally, we see that all noetherian rings are contained in $\mathcal{K}_{2}(\mathcal{D}, \unlhd)$. For if we suppose $A$ to be a ring not in $\mathcal{K}_{2}(\mathcal{D}, \unlhd)$, there must be an ideal $I$ of $A$ such that $I \subseteq \mathcal{D}(A)$ and $I \notin \mathcal{D}$. Choose $i \in I, p$ prime such that $p x \neq i$ for all $x \in I$; then $p I \neq I$, and $(A / I)[p] \neq 0$ (where $(A / I)[p]$ is defined in the same way as $K[p]$ above) since there must be an element $d$ of $\mathcal{D}(A)$ wiht $p d=i$. As $(A / I)[p] \unlhd A / I$, there is an ideal $J$ of $A$ with $J / I=(A / I)[p]$ and satisfying $p J \subseteq I \varsubsetneqq J$ (as $d \in J \backslash I$ ). Now $(A / I)[p] \cap \mathcal{D}(A) / I=\{c+I \mid c \in \mathcal{D}(A) \& p c \in I\} \neq 0$ since $d \notin I, d \in \mathcal{D}(A)$ and $p d=i \in I$, so $I \varsubsetneqq J \cap \mathcal{D}(A)$. Also $J \cap \mathcal{D}(A) \varsubsetneqq \mathcal{D}(A)$, as otherwise $\mathcal{D}(A) \subseteq J$, so $p \mathcal{D}(A) \subseteq I$-a contradiction, as $p \mathcal{D}(A)=\mathcal{D}(A)$ and $I \varsubsetneqq \mathcal{D}(A)$.

But now $p(J \cap \mathcal{D}(A)) \neq J \cap \mathcal{D}(A)$ (since if $p(J \cap \mathcal{D}(A))=J \cap \mathcal{D}(A)$ we would have $J \cap \mathcal{D}(A) \subseteq p J \subseteq I)$, so $J \cap \mathcal{D}(A) \neq \mathcal{D}(J \cap \mathcal{D}(A))$.

In a similar way, we can find $K \unlhd A$ with $J \cap \mathcal{D}(A) \varsubsetneqq K \cap \mathcal{D}(A), K \cap \mathcal{D}(A) \neq$ $\mathcal{D}(K \cap \mathcal{D}(A))$ and so on, giving an infinite ascending chain of ideals of $A$. Hence $A$ is not noetherian.

From these last two facts we get the following:

Proposition 4.1. Let $A$ be a noetherian ring. Then $\mathcal{D}(A)$ is a $Q$-algebra, and for any ideal $I$ of $A, I \cap \mathcal{D}(A)$ is an algebra ideal of $\mathcal{D}(A)$.

Corollary 4.2. If $A$ is a divisible noetherian ring, then $A$ is a $Q$-algebra, and every ring ideal of $A$ is an algebra ideal. 
For our second example, we look at the Jacobson radical, and introduce another class of interest,

$$
\mathcal{K}_{0}(\mathcal{J})=\{A \mid a \in \mathcal{J}(A) \text { whenever } a \text { is quasiregular }\},
$$

where $a$ is quasiregular in $A$ if $a \circ b=a+b-a b$ for some $b \in A$. If we let $\mathcal{S}$ be the semisimple class of the Jacobson radical, then $\mathcal{S} \cap \mathcal{K}_{0}(\mathcal{J})$ is the class of rings without non-zero quasiregular elements, and we have

\section{PROPOSITION 4.3.}

$\mathcal{S} \cap \mathcal{K}_{0}(\mathcal{J})$ is a semisimple class.

Proof: Let $A$ be a ring, $I$ an ideal of $A$ with $I$ and $A / I \in \mathcal{S} \cap \mathcal{K}_{0}(\mathcal{J})$, and let $a, b \in A$ with $a \circ b=0$. Then $(a+I) \circ(b+I)=0$, so $a, b \in I$, as $A / I \in \mathcal{S} \cap \mathcal{K}_{0}(\mathcal{J})$. But then $a=b=0$, since $I \in \mathcal{S} \cap \mathcal{K}_{0}(\mathcal{J})$. That is, $A \in \mathcal{S} \cap \mathcal{K}_{0}(\mathcal{J})$, so $\mathcal{S} \cap \mathcal{K}_{0}(\mathcal{J})$ is closed under extensions.

Let $\left\{A_{\lambda} \mid \lambda \in \Lambda\right\}$ be a family of rings in $\mathcal{S} \cap \mathcal{K}_{0}(\mathcal{J})$, and let $\prod A_{\lambda}$ denote their direct product. Let $\left(a_{\lambda}\right)_{\Lambda},\left(b_{\lambda}\right)_{\Lambda}$ be elements of $\prod A_{\lambda}$ with $\left(a_{\lambda}\right)_{\Lambda} \circ\left(b_{\lambda}\right)_{\Lambda}=0$. Then $a_{\lambda} \circ b_{\lambda}=0$ for every $\lambda$, so $a_{\lambda}=b_{\lambda}=0$ for every $\lambda$ since each $A_{\lambda}$ is in $\mathcal{S} \cap \mathcal{K}_{0}(\mathcal{J})$, and so $\mathcal{S} \cap \mathcal{K}_{0}(\mathcal{J})$ is closed under direct products. Since $\mathcal{S} \cap \mathcal{K}_{0}(\mathcal{J})$ is clearly closed under subrings, and hence under subdirect products and ideals, the result follows.

We will denote the radical class corresponding to $\mathcal{K}_{0}(\mathcal{J}) \cap \mathcal{S}$ by $\mathcal{Q}$.

PROPOSITION 4.4. Let $R$ be a ring. Then $R \in \mathcal{K}_{0}(\mathcal{J})$ if and only if $R$ is an extension of a Jacobson radical ring by a ring of $\mathcal{K}_{0}(\mathcal{J}) \cap \mathcal{S}$.

Proof: Suppose $\mathcal{J}(R)$ is not the set of quasiregular elements of $R$, and let $a \epsilon$ $R \backslash \mathcal{J}(R), a \circ b=0$. Then $b \in R \backslash \mathcal{J}(R)$, and $(a+\mathcal{J}(R)) \circ(b+\mathcal{J}(R))=a \circ b+\mathcal{J}(R)=0$, so $0 \neq a+\mathcal{J}(R)$ is a quasiregular element of $R / \mathcal{J}(R)$, and $R / \mathcal{J}(R) \notin \mathcal{S} \cap \mathcal{K}_{0}(\mathcal{J})$.

Conversely, let $(a+\mathcal{J}(R)) \circ(b+\mathcal{J}(R))=0$ in $R / \mathcal{J}(R), a \notin \mathcal{J}(R)$. Then $a \circ b \in$ $\mathcal{J}(R)$, so there is an element $c$ of $\mathcal{J}(R)$ with $(a \circ b) \circ c=0$. But then $a \circ(b \circ c)=0$, so a is quasiregular in $R$. Hence $\mathcal{J}(R)$ is not the set of quasiregular elements of $R$. D

Now, let $S \dashv R$ mean that $S$ is a subring of $R$, and let $S<R$ mean that $S$ is a left ideal of $R$. We look at the relationship between $\mathcal{K}_{0}(\mathcal{J}), \mathcal{K}_{1}(\mathcal{J},-1), \mathcal{K}_{2}(\mathcal{J},-1)$ and $\mathcal{K}_{2}(\mathcal{J},<)$ (noting that $\mathcal{K}_{1}(\mathcal{J},<)$ is the class of all rings. By [10, Theorem 2] a ring $R$ of the form $\left[\begin{array}{ll}\Delta & Y \\ X & \Gamma\end{array}\right]$, where $\Delta$ and $\Gamma$ are division rings, $Y$ is a $\Delta \Gamma$-bimodule, $X$ is a $\Gamma \Delta$-bimodule and

$$
\left[\begin{array}{ll}
a & y \\
x & b
\end{array}\right]\left[\begin{array}{cc}
c & z \\
w & d
\end{array}\right]=\left[\begin{array}{cc}
a c & z+y d \\
x c+b w & b d
\end{array}\right]
$$


(and addition is componentwise), has the property that its Jacobson radical is the set of non-regular elements. The regular elements are of the form $\left[\begin{array}{ll}a & y \\ x & b\end{array}\right],\left[\begin{array}{ll}a & y \\ x & 0\end{array}\right]$ or $\left[\begin{array}{ll}0 & y \\ x & b\end{array}\right]$ where $a \in \Delta, b \in \Gamma$ are non-zero, $x \in X, y \in Y$ and elements of the form $\left[\begin{array}{ll}0 & y \\ x & 0\end{array}\right]$ are nilpotent. To demonstrate that this property is passed on to a left ideal $L$, it suffices to show that every non-nilpotent element of $L$ is regular, since the nilpotent elements of $L$ clearly form an ideal and a non-zero regular element cannot be contained in the Jacobson radical. If $\left[\begin{array}{ll}a & y \\ x & b\end{array}\right] \in L$, then

$$
\begin{gathered}
{\left[\begin{array}{cc}
a^{-2} & -a^{-2} y b^{-1}-a^{-1} y b^{-2} \\
-b^{-2} x a^{-1}-b^{-1} x a^{-2} & b^{-2}
\end{array}\right]\left[\begin{array}{ll}
a & y \\
x & b
\end{array}\right]} \\
=\left[\begin{array}{cc}
a^{-1} & -a^{-1} y b^{-1} \\
-b^{-1} x a^{-1} & b^{-1}
\end{array}\right] \in L
\end{gathered}
$$

and $\quad\left[\begin{array}{ll}a & y \\ x & b\end{array}\right]\left[\begin{array}{cc}a^{-1} & -a^{-1} y b^{-1} \\ -b^{-1} x a^{-1} & b^{-1}\end{array}\right]\left[\begin{array}{ll}a & y \\ x & b\end{array}\right]=\left[\begin{array}{ll}a & y \\ x & b\end{array}\right]$

so $\left[\begin{array}{ll}a & y \\ x & b\end{array}\right]$ is regular in $L$, and if $\left[\begin{array}{ll}a & y \\ x & 0\end{array}\right] \in L$, then

$$
\left[\begin{array}{cc}
a^{-2} & 0 \\
0 & 0
\end{array}\right]\left[\begin{array}{ll}
a & y \\
x & 0
\end{array}\right]=\left[\begin{array}{cc}
a^{-1} & a^{-2} y \\
0 & 0
\end{array}\right] \in L
$$

and

$$
\left[\begin{array}{ll}
a & y \\
x & 0
\end{array}\right]\left[\begin{array}{cc}
a^{-1} & a^{-2} y \\
0 & 0
\end{array}\right]\left[\begin{array}{ll}
a & y \\
x & 0
\end{array}\right]=\left[\begin{array}{ll}
a & y \\
x & 0
\end{array}\right]
$$

so $\left[\begin{array}{ll}a & y \\ x & 0\end{array}\right]$ is regular in $L$. Similarly, $\left[\begin{array}{ll}0 & y \\ x & b\end{array}\right]$ is regular in $L$, so the Jacobson radical of any such left ideal $L$ is the set of non-regular elements, as every element is regular or quasiregular. This means that $\mathcal{J}(L)$ is the set of nilpotent elements of $L$, and thus $\mathcal{J}(L)=L \cap \mathcal{J}(R)$. That is, $R \in \mathcal{K}_{2}(\mathcal{J},<)$ for any such $R$.

We also see that an element $\left[\begin{array}{ll}a & 0 \\ 0 & b\end{array}\right]$ of such a ring $R$ is quasiregular whenever $a$ and $b$ are quasiregular. Thus any subring $S$ of diagonal matrices not containing $\left[\begin{array}{ll}1 & 0 \\ 0 & 0\end{array}\right],\left[\begin{array}{ll}0 & 0 \\ 0 & 1\end{array}\right]$ or $\left[\begin{array}{ll}1 & 0 \\ 0 & 1\end{array}\right]$ is quasiregular, so that $\mathcal{J}(S) \neq S \cap \mathcal{J}(R)$ and in fact, $S \nsubseteq \mathcal{J}(R)$; that is, $R \notin \mathcal{K}_{1}(\mathcal{J},-1)$ (or $\mathcal{K}_{2}(\mathcal{J},-1)$ ).

The prime fields $Z_{p}$, since they have no non-trivial subrings are in $\mathcal{K}_{2}(\mathcal{R},-1)$ for any $\mathcal{R}$, but every element besides 1 is quasiregular in $Z_{p}$, so that $Z_{p} \notin \mathcal{K}_{0}(\mathcal{J})$ for $p>2$. 
Let $A=\{m / n \mid m, n \in \mathbf{Z}, \quad n \notin 2 Z\} ;$ then $\mathcal{J}(A)=\{m / n \mid m \in 2 Z, \quad n \notin 2 Z\}$. Thus if $a$ is quasiregular in $A, a+\mathcal{J}(A)$ is quasiregular in $A / \mathcal{J}(A) \cong \mathbf{Z}_{2}$, so $a+\mathcal{J}(A)=$ 0. Thus $a \in \mathcal{J}(A)$, and so $A \in \mathcal{K}_{0}(\mathcal{J})$. However, $Z \dashv A$, and $Z \cap \mathcal{J}(A)=2 Z$ while $\mathcal{J}(\mathbf{Z})=0$, so $A \notin \mathcal{K}_{2}(\mathcal{J},-1)$.

To sum up the relationships among these classes, we have $\mathcal{K}_{0}(\mathcal{J}) \varsubsetneqq \mathcal{K}_{2}(\mathcal{J},<)$; $\mathcal{K}_{2}(\mathcal{J},-1) \varsubsetneqq \mathcal{K}_{2}(\mathcal{J},<) ; \mathcal{K}_{2}(\mathcal{J},-1) \varsubsetneqq \mathcal{K}_{1}(\mathcal{J},-1) ; \mathcal{K}_{0}(\mathcal{J}) \varsubsetneqq \mathcal{K}_{1}(\mathcal{J},-1) ; \mathcal{K}_{2}(\mathcal{J},<) \varsubsetneqq$ $\mathcal{K}_{1}(\mathcal{J},-1)$; and $\mathcal{K}_{0}(\mathcal{J}) \neq \mathcal{K}_{2}(\mathcal{J},-1)$, where neither class is contained in the other. The only question remaining is whether or not $\mathcal{K}_{1}(\mathcal{J},-1)$ is contained in $\mathcal{K}_{2}(\mathcal{J},<)$.

By Proposition 1.5 and the remarks before it, $\overline{\mathcal{K}}_{2}(\mathcal{J},-1)=\overline{\mathcal{K}}_{1}(\mathcal{J},-1)$ is a semisimple class properly contained in $\mathcal{S}$, and from the previous discussion $\mathcal{K}_{0}(\mathcal{J}) \cap \mathcal{S}$ is a semisimple class properly contained in $\overline{\mathcal{K}}_{1}(\mathcal{J},-1)$. Thus

$$
\mathcal{J} \varsubsetneqq \mathcal{J}_{\dashv} \varsubsetneqq \mathcal{Q} \text {. }
$$

It is interesting to note that not every $\mathcal{J}_{\mathcal{H}}$-semisimple ring is a subdirect product of primitive $\mathcal{J}_{\dashv}$-semisimple rings, a counter-example being provided in [2].

It might be worthwhile to work with a similar class to $\mathcal{K}_{0}(\mathcal{J})$ for other radical classes which can be defined in terms of elements.

NOTE ADDED IN PROOF.

In a recent paper ("General Heredity and Strength for Radical Classes", Can. J. Math.), 40(6) (1988), 1410-1421) A.D. Sands and P.N. Stewart have given a characterisation like Theorem 2.5 for a more general type of subring than those considered here. Their approach involves a transfinite process to construct the appropriate semisimple class.

\section{REFERENCES}

[1] V.A. Andrunakievic, 'Radicals of associative rings I', Amer. Math. Soc. Transl. (2) 52 (1966), 95-128.

[2] V.A. Andrunakievich and Yu. M. Ryabukhin, 'Algebras without quasiregular subalgebras', Soviet Math. Dokl. 29 (1984), 234-237.

[3] R. Baer, 'Meta ideals', Report of a Conference on Linear Algebras, Publication 502, Nat. Acad. Sci.-Nat. Res. Council, Washington D.C., (1957), 33-52.

[4] N. Divinsky, Rings and Radicals (Allen and Unwin, 1965).

[5] L. Fuchs, Infinite Abelian Groups, Volume II (Academic Press, New York, 1973).

[6] B.J. Gardner, 'Some radical constructions for associative rings', J. Austral. Math. Soc. 18 (1974), 442-446.

[7] B.J. Gardner, 'Radicals and left ideals', Bull. Acad. Polon. Sci. Ser. Sci. Math. Astronom. Phys. 24 (1974), 359-365. 
[8] J. Krempa and E. Stankiewicz, 'Radicals of meta ideals', Bull. Acad. Polon. Sci., Ser. Sci. Math. Astronom. Phys. 22 (1974), 359-365.

[9] S. Lajos and F. Szasz, 'On $(m, n)$-ideals in associative rings', Publ. Math. Debrecen. 25 (1978), 265-273.

[10] W.K. Nicholson, 'Rings whose elements are quasi-regular or regular', Aequationes Math. 9 (1973), 64-70.

[11] E. Puczylowski, 'A note on hereditary radicals', Acta. Sci. Math. (Szeged) 44 (1982), 133-135.

[12] P.N. Stewart, 'Strict radical classes of associative rings', Proc. Amer. Math. Soc. 39 (1973), 273-278.

[13] T. Szele, 'Zur Theorie der Zeroringe', Math. Ann. 121 (1949), 242-246.

[14] R. Wiegandt, 'Radical and semisimple classes of rings', Queen's Papers in Pure and Applied Mathematics (Kingston, Ontario) 37 (1974).

Department of Mathematics

University of Tasmania

GPO Box 252C

Hobart TAS 7001

Australia 
